Original Research Paper

\title{
Markov-Modulated Bernoulli-Based Performance Analysis for Gentle BLUE and BLUE Algorithms under Bursty and Correlated Traffic
}

\author{
${ }^{1}$ Adeeb Alsaaidah, ${ }^{1}$ Mohd Zalisham, ${ }^{1}$ Mohd Fadzli and ${ }^{2}$ Hussein Abdel-Jaber \\ ${ }^{1}$ Faculty of Science and Technology, Universiti Sains Islam Malaysia (USIM), Nilai, Malaysia \\ ${ }^{2}$ Faculty of Computer Studies, Arab Open University, Kingdom of Saudi Arabia
}

\author{
Article history \\ Received: 23-08-2015 \\ Revised: 27-06-2016 \\ Accepted: 28-06-2016 \\ Corresponding Author: \\ Adeeb Alsaaidah \\ Faculty of Science and \\ Technology, Universiti Sains \\ Islam Malaysia (USIM), Nilai, \\ Malaysia \\ Email:Adeeb1986@yahoo.com
}

\begin{abstract}
This paper presents a performance study for Gentle BLUE (GB) under the bursty and correlated properties of aggregated network traffic. The Bernoulli Process (BP) fails to represent the properties of aggregated correlated and bursty traffic, so instead of that, MMBP has been used. MMBP is A 2D discrete-time Markov chain modeling for GB algorithm with two traffic classes, each with its own parameters. The proposed model is compared with the GB that uses the BP as a source model (GB-BP) and original BLUE that uses the BP (BLUE-BP) and MMBP (BLUE-MMBP-2) as source model. The evaluation is conducted in term of queuing waiting time, mean queue length, throughput, packet loss and dropping probability. When congestion (e.g., heavy congestion) occurs, the results show that GBMMBP-2 provides the bestmean queue length, queuing time and packet loss among the compared methods.
\end{abstract}

Keywords: Congestion Control, Queue Management, Markov Modulated Bernoulli Process, Gentle BLUE, Performance Evaluation

\section{Introduction}

Due to the enormous developments of internet technology in numerous applications such as, audio and video data traffic, a high speed router buffers is a demand. The packets pass through different router until reach their destination. Thus, each router buffer receives packets from several traffic classes with different aggressive aspect. Aggressive traffic can increase the number of packet arrival at the router buffer, which subsequently declines the performance of network by decreasing the throughput and raising both, packet loss rate and queuing delay (Reddy and Ahammed, 2008; Baklizi et al., 2014), as a result of a congestion problem. Congestion is defined as an event, which happens in networks, when the network resources are not able to accommodate the number of inserted packets (Ryu et al., 2003; Baklizi et al., 2013).

Various algorithms, such as Drop Tail (DT), DT is the conventional control technique, has been proposed to control the congestion and enhance the network performance. DT handles congestion at the network router in a late stage (after the router buffer overflows). For the purpose of overcoming these disadvantages in DT, Active Queue Management (AQM) algorithms have been developed. Examples of AQM algorithms are, BLUE (Feng et al., 2002), Random Early Detection (RED) (Floyd and Jacobson, 1993), dynamic Gentle Random Early Detection DGRED (Baklizi et al., 2013), adaptive RED (Floyd et al., 2001), Distortion Constrained (DC) (Ghoreishi et al., 2015), Enhancement of Fair Random Early Detection Algorithm (EFRED) (Abdulkareem et al., 2015) and Gentle BLUE (GB) (Alsaaidah et al., 2014).

The key issues of the AQM algorithm performance evaluation are queue modeling and parameter tuning. The ultimate goal of using queue modeling is to evaluate or validate the performance. Models, such as the renewal traffic (Bernoulli Process (BP), Poisson Processes (PPs)) and Markov modulate arrival such as Markov-Modulated Bernoulli Arrival Process (MMBP) (Lim et al., 2010; Alsaaidah et al., 2014), can be used. Several AQM algorithms have been evaluated based on $\mathrm{BP}$, which is a discrete time process that deals with network traffic as a single class. However, network traffic comes in different classes that the Bernoulli process cannot in efficient manner. This is because Bernoulli is unable to represent aggregated traffic, such as multimedia, which has a two important traffic aspect bursty and correlated (Samorodnitsky, 2007). 
Burstiness" is observable in the BP through the clustering phenomenon of arrival packets in the timeline" (Liu et al., 2008). The long-range dependency produced by high correlated between the inter-arrival times are not handled using BP, which gives a false evaluation results (Liu et al., 2008).

In order to get a good network performance, the router buffer should be managed. Congestion control method, like AQMs, should be applied to manage router buffers. The properties of packet arrival traffic, such as burstiness and correlation, should also be considered as the input in the process of the evaluation of AQM algorithms. Thus, this paper proposes an AQM method and atraffic modeling. This study uses a two-state MMBP (MMBP-2) to model the queuing process and deal with the properties of burstiness and correlations.

This paper presents a discrete-time performance analysis on GB using MMBP-2 as the traffic source. Furthermore, we experimentally compare the proposed GB-that use both BP and MMBP-2 as a source model and the original BLUE that uses BP (BLUE-BP) and MMBP (BLUE-MMBP-2) as source models. This comparison shows which of the compared algorithm offers more satisfactory performance under bursty and correlated traffics. The performance evaluation is implemented by analyzing the network measuresunder two traffic classes using (MMBP-2) and under one traffic class using BP.

Section 2 presents the related work;section 3 and section 4\% the BLUE and Gentle BLUE algorithms; The two state Markov-modulated Bernoulli processes is introduced at section 5; section 6 introduces the procedure that is used to model the arriving traffic based on MMBP-2; the simulation details is highlighted at section 7 ; section 8 demonstrates the results and section 9 provides a conclusion.

\section{Related Work}

Developing a system model is an essential way to deal with the system requirements that gives a good prediction of the system performance. The optimal evaluation of the effective networks congestion control techniques relied on modeling system that characterize the features and characteristics of network traffics in order to guarantee the reliability and affordability. Modeling traffic plays important roles in the evaluating process. Renewal traffic (PP and BP) are traditional modeling approaches that are based on simple mathematical calculation (Lee et al., 1997). These traditional approaches are unable to represent the aggregated traffic, or in other word, unable to capture the properties of the aggregation such as correlation and burstiness ( $\mathrm{Ng}$ et al., 1999). Renewal traffic limitation is defeated using Markov-Modulated Arrival
(MMA). There is three types of MMA that are used to model arrival packets, these are: Markov-Modulated Fluid Flow (MMFF), Markov-Modulated Poisson Processes (MMPP) and MMBP (Ng et al., 1999). MMA models represent both discrete and continuous time scale and packet arrival. Only a single event allowed for the packet at any time (the packet may be arrive or departure). While in continuous approach, at contrary, multiple events could be occurred for the packet at a single time slot (the packet may arrive and/or departure) indiscrete time queue (Al-Diabat et al., 2012; Abdel-Jaber et al., 2007a).

The types of MMA based on time scale and packet arrival, are summarized in Table 1.

MMFF is used to represent the characteristics of continuous approach in both packet arrival and time scale (Ng et al., 1999). On other hand, MMPP (Fischer and Meier-Hellstern, 1993) is widely used in modeling applications that hasdiscrete packet arrival with continuous time (Lim et al., 2011). The computer and communication are discrete in nature, which means MMPP and MMFF does not capture the characteristics that represent the digitalized communications (Lim et al., 2011). As a result, MMBP is suitable candidate for modeling nature traffic due to the ability to model the traffic using discrete time approach with bursty and correlated traffics properties (Lim et al., 2011; Ng et al., 1999; Guan et al., 2006; Kim et al., 2010; Lim et al., 2009; Guan et al., 2004a).

MMBP is equivalent to MMPP but in discrete-time ( $\mathrm{Ng}$ et al., 1999). Numerous researches model their arrival traffic by using MMBP. In particular (Lim et al., 2009; Guan et al., 2006) propose an approach to control the delay on a router at specific level under aggregated Internet traffic flows using MMBP. Similarly, the work in (Guan et al., 2004b) evaluates RED under bursty and correlated traffic using MMBP. The evaluation method in (Lim et al., 2010) valuates RED and WRED using MMBP-2. Active-WRED is proposed in (Kim et al., 2010) and uses MMBP to model its arrival process.

In summary, as internet traffic is aggregated in natural so modeling these traffic need an approach that capture discretized, bursty and correlation for the traffics, so MMBP is a suitable candidate to represent these all characteristic. By contrast, MMPP and MMFF unable to capture this characteristic especially correlated and bursty traffic ( $\mathrm{Ng}$ et al., 1999; Lim et al., 2011; 2010; 2009).

Table 1. MMA categories

\begin{tabular}{llll}
\hline MMA & MMFF & MMPP & MMBP \\
\hline Time scale & Continuous & Continuous & Discrete \\
Packet arrival & Continuous & Discrete & Discrete \\
\hline
\end{tabular}




\section{BLUE Algorithm}

BLUE algorithm (Feng et al., 2002) rely on DP and a single threshold to control congestion, BLUE increases DP if the queue size at the router buffer above the threshold, while it decrease DP if the queue size at the router buffer is empty or the link is idle. BLUE algorithm use fixed rate for increasing or decreasing DP.

BLUE usesfew parameters to control the value of DP. The parameter f_times represents the minimum allowed period between two subsequent DP adjustments. The parameter Pin represents the amount of DP incremented. The parameter Pde represents the amount of DP decremented. The DP calculation at BLUE algorithm is shown in algorithm 1:

\section{Algorithm 1: Dropping Probability in the BLUE} Algorithm

1. Begin

2. if (buff_le $>$ th): Plos

3. if (time _of the 1 adj $>f$ _time)

4. $\mathrm{D}_{\mathrm{P}}=\mathrm{D}_{\mathrm{P}}+\mathrm{Pin}$

5. $\mathrm{L}_{-}$adj $=$current

6. if (que buff $==0$ ) // (buffer is empty)

7. $\mathrm{D}_{\mathrm{P}}=\overline{\mathrm{D}_{\mathrm{P}}}-\mathrm{Pde}$

8. $\quad \mathrm{L} \_$adj $=$current

9. End

10. Where the que buff $=$ queue buffer

11. buff_le $=$ buffer length

12. $\mathrm{Th}=$ threshold

13. plos $=$ packet losing

14. ladj $=$ last adjustment

15. time of the ladj=time of the last adjustment

16. pin $=$ pincrementpde $=$ pdecrement

In lines 2 to 4, DP increases by the value of $P$ in if the of number of packet in the buffer is greater than the threshold value and the last adjustment is outdated. The last adjustment time is updated in line 5. On the other hand, if the buffer is empty, DP is decreased by the value of Pdein lines 6 to 8 and the last adjustment time is updated in line 8 .

\section{Gentle BLUE Algorithm}

GB (Alsaaidah et al., 2014) extends the well-known BLUE algorithm (Feng et al., 2002) by providing a dynamic mechanism to calculate DP value based on the queue length status. GB is proposed with the response to the limitation of BLUE algorithm.As such, BLUE algorithm calculates DP using a fixed value while GB calculates the DP dynamically. Moreover, GBreduces the problem of parameter setting. This dynamic mechanism that updates the DP value depends on the parameters $\mathrm{q}$ and a single threshold. DP of the GB algorithm is provided in Algorithm 2:

\section{Algorithm 2: $D_{P}$ in the GB Algorithm}

1. Begin

2. if $\left(\mathrm{q} \_\right.$th $>0 \& \& \mathrm{q} \_$th $\left.<=\mathrm{Th}\right)$

3. $\{$ if (C_time of the 1 ajd $>f$ _time $)\{$

4. $\left.D P=\overline{\left(\mathrm{q} \_ \text {th } / \overline{\mathrm{T}} \mathrm{h}\right.}\right) *((\overline{\mathrm{C}}-\mathrm{Th}) / \mathrm{C}) *(1$-init $D P)$

5. $\mathrm{L}_{\mathrm{adj}}=$ current $\}$

6. Else $\{$ if $(q$ th $>$ Th\&\&q th $<\mathrm{C})\{$

7. $D P=((\mathrm{C}-\mathrm{Th}) / \mathrm{C}) *(1-$ init $D P)+\left(\mathrm{q} \_\right.$th $\left./ \mathrm{C}\right) *(1-((\mathrm{C}-$

Th)/C) *(1-init $D P)$

8. $\quad L_{-}$adj $=$current $\}$\}

9. Else

10. $\{D P=1 / /$ means all arrival packet will be dropped

11. $L_{-}$adj $=$current $\}$

12. End

13. whereq th: Queue length

14. Th: Threshold

15. c c time of the 1_adj: Current time of the last adjustment

16. initDP:InitialDP

17. L_adj: Last adjustment

18. C: Capacity

19. f_time: Freeze time

In lines 2 to 5, DP adjustment is implemented when no congestion occurs. In this case, DP is calculated as given in line 4. In lines 6 to 8 , DP adjustment is implemented in case of light and heavy congestion. GB algorithm uses the equation in line 7 to calculate DP. In lines 9 to 11, DP is set up when the number of packet at the router buffer is greater than the size of the router buffer. All arriving packet will be dropped and the value of DP is equal to 1 .

\section{Bernoulli Process}

BP is formulate by a sequence of random binary variablesYi. BP arrivals can only occur at a time slot $\mathrm{k}$. The probability of the arrival $\mathrm{p}$ in the time slot $\mathrm{k}$ is independent from any other arrivals. The arrival in slot $\mathrm{k}$ is binomially distributed (Woodward, 1994; Abdel-Jaber et al., 2007b) the two possible values of each Yi are $\mathrm{p}$ and (1-p), where $p$ is the successful probability. The number of time slots between two arrivals is geometrically distributed with parameter p (Abdel-Jaber et al., 2007b).

\section{Source Model (MMBP-2)}

The MMBP is used for modeling GB algorithm in bursty and correlated traffic. MMBP could be extended to contain N-state but for simplicity analysis, presentation and discussion, two states have been used $(\mathrm{Ng}$ et al., 1999). The proposed model is shown in Fig. 1.

MMBP-2 has two states with distinct packet arrival probability, $\alpha 0$ and $\alpha 1$. MMBP uses discrete time queue, which means the time is divided into slots as similar to Bernoulli; however, there is no independency of arrivals. 


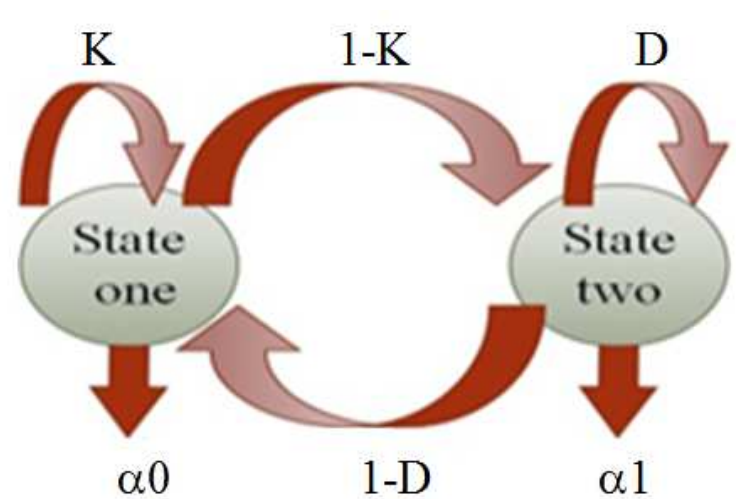

Fig. 1. MMBP-2

Assume that the packet is arrived to a state one in time slot $\mathrm{k}$, the next packet can remains in the same state, with probability $\mathrm{D}$ or transited to the next state, with probability 1-D. The difference between BP and MMBP2 is that MMBP-2 uses two stateswhile BP uses one state, represented by a transition probability matrix TPm for its process and diagonal matrix DMp for the arrival probabilities as follows (Guan et al., 2007):

$$
T P m=\left[\begin{array}{cc}
K & 1-K \\
1-D & D
\end{array}\right]
$$

And:

$$
D M p=\left[\begin{array}{cc}
\alpha 0 & 0 \\
0 & \alpha 1
\end{array}\right]
$$

According to MMBP-2, the steady state probabilities for state one $\left(S \_S P(S 1)\right)$ and state two $\left(S \_S P(S 2)\right)$ can be represent as in "Equation 1 and 2":

$$
\begin{aligned}
& S_{-} S P(S 1)=(K) S_{-} S P(S I)+(1-D) S_{-} S P(S 2) \\
& S_{-} S P(S 2)=(1-K) S_{-} S P(S 1)+D S_{-} S P(S 2)
\end{aligned}
$$

where, $S \_S P(S 1)$ and $S \_S P(S 2)$ denote the steady-state probabilities of $\mathrm{MMBP}-2$ in state 1 and state 2 respectively.

\section{Modeling GB Arrival Traffic using MMBP}

GB has been modeling by using MMBP-2 in this study. MMBP-2 has two state and each one has its own parameters. In state one the probability of packet arrival is denoted by $\alpha 0$, whereas in state 2 , is denoted by $\alpha 1$. In both states, DP remains the same as the original GB. Figure 2 illustrates the steps of the proposed GBMMBP-2 algorithm.

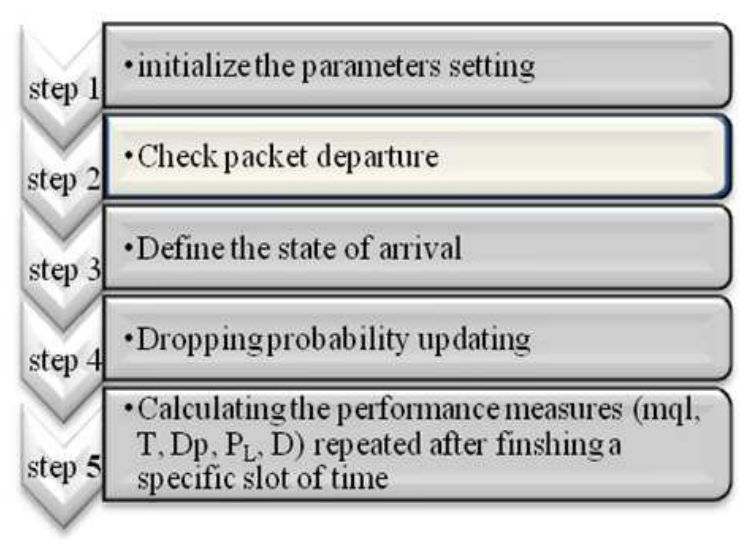

Fig. 2. GB using MMBP-2

Step one (initialize the parameters setting): GBMMBP-2 required settings of many parameters as seen in Table 2. GB usesa threshold value as $60 \%$ of the buffer size as those in (Saaidah et al., 2014; Abdel-Jaber et al., 2007a; Al-Diabat et al., 2012). Initially, the queue length is empty, which means the initial value of queue length is zero and the packet will be arrived initially to state 1 , thus the initial state is state 1. The initial DP (initDP) parameter is set to a value similar to the original BLUE (Feng et al., 2002) and original GB (Alsaaidah et al., 2014).

Step two (check packet departure): The departure comes before the arrival in this discrete-time queue. If a packet departed, then the queue length is decreased by one, else the ql remains unchanged. The queue length is calculated in GB-MMBP-2 using the arrival packets. At the beginning, the queue length of the router buffer will beempty and increased by one with every arrived packet.

Step three (define the state of arrival): GB-MMBP-2 is consist of two states, a high priority to the newly arriving packet to remain in the same state is giving with probability 0.9 and less priority for transition to another state with probability of 0.1 . The probabilities of states themselves are equal. The decision of packet hosting to state one or state two is based on the packet arrival probability for that state. Figure 3 illustrates the definition of the state for an arrival packet.

Step four (DP updating): GB-MMBP-2 dynamically updates DP using the same updating mechanism as GB algorithm for both states (state 1 and state 2).

Step five (calculating performance measure): The performance measures for each algorithm are collecting after the algorithm executed 10 times with different seeds. Seeds are required in the generation of the random number. The number of slots used in the experiments is $2,000,000$. In each seed, the performance measures (mql, D, T, DP, PL) are calculated after the simulation reach a steady state. The mean of these runs at a specific packet arrival probability are then collected. 


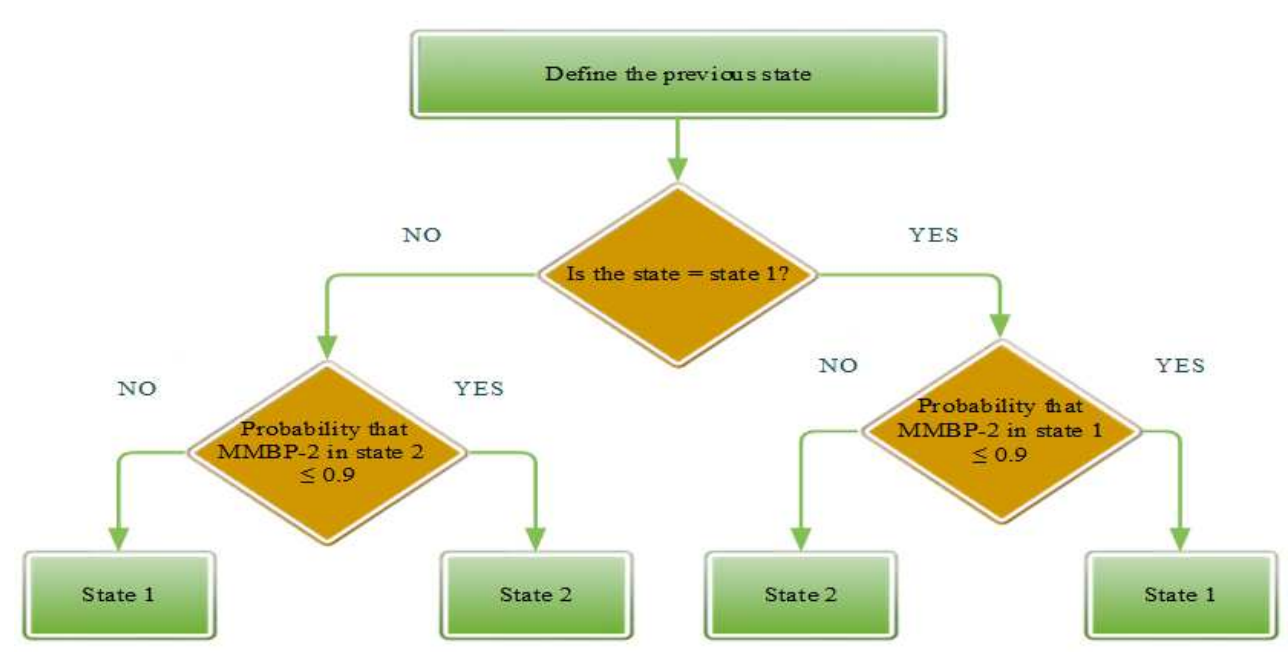

Fig. 3. Defining the arrival packet state

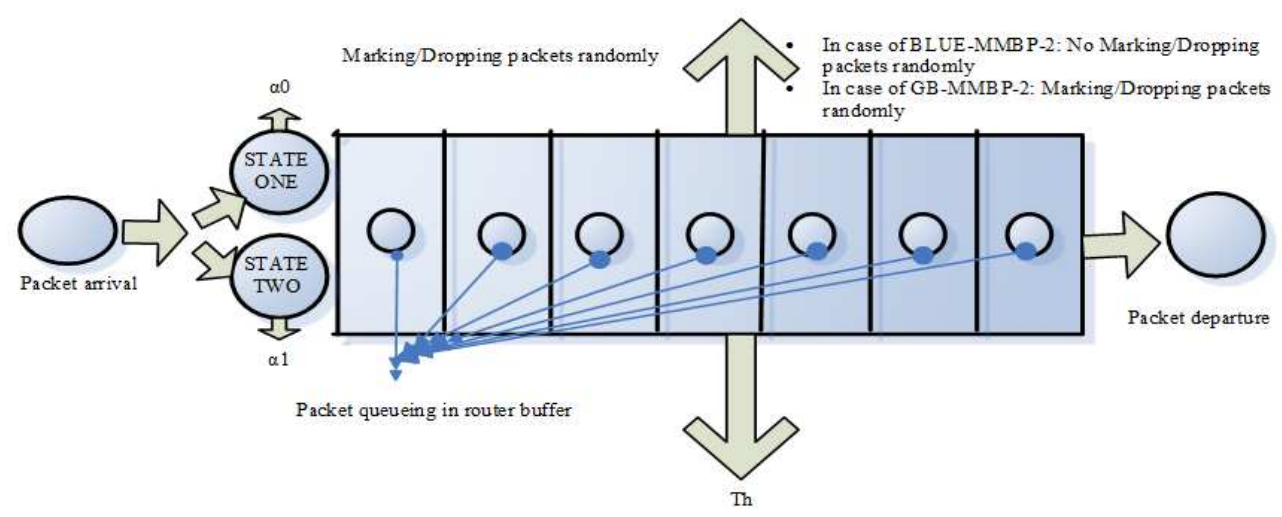

Fig. 4. Single router buffer for BLUE-MMBP-2 and GB-MMBP-2

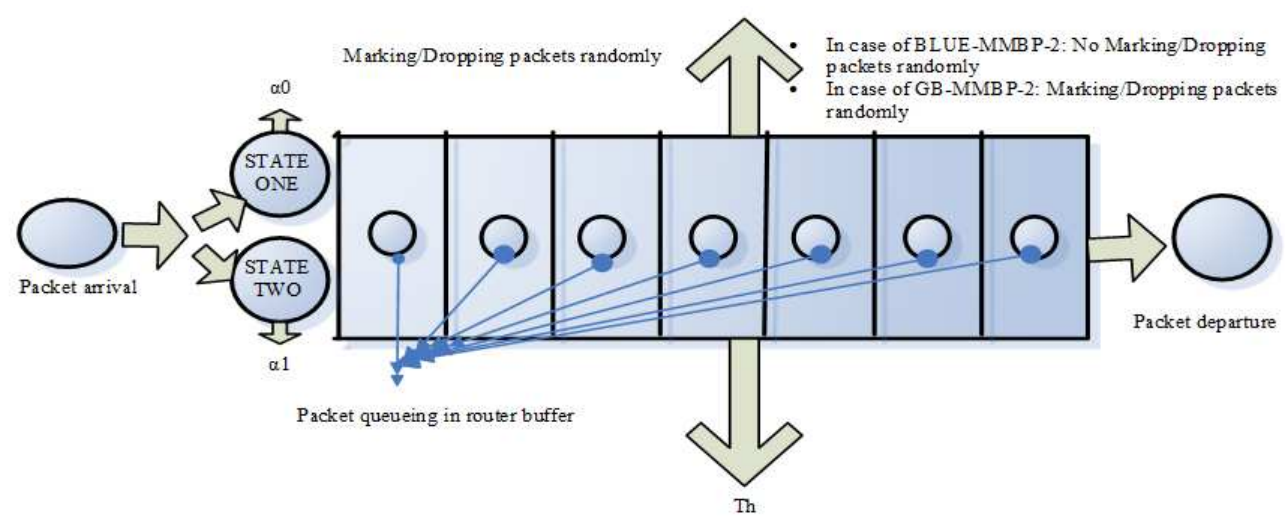

Fig. 5. Single router buffer for BLUE-BP and GB-BP

\section{Simulation}

In this section, GB-MMBP-2, BLUE-MMBP-2, GB$\mathrm{BP}$ and BLUE-BP are simulated. A discrete-time queue approach used to model the queue. In this approach, the simulation period is divided into time units called slot. In each slot, multiple events could be occurred (Abdel-Jaber et al., 2015; 2008; Zhou and Wang, 2008; Guan et al., 2004c). The probability of packet arrival in BLUE-BP and GB-BP, is denoted by $\alpha 0$, while the packet departure probability in a slot is called by $\beta$. As for the probability of packet arrival in GB-MMBP-2 and 
BLUE-MMBP-2, for state 1 and state 2 are called by $\alpha 0$ and $\alpha 1$, respectively. The probability of packet departure in a slot for all simulated algorithm called by $\beta$. A geometrical distribution is used to model the packet departure. Packet inter-arrival times are geometrically distributed with a mean (1/ $\alpha 0$ for BLUE-BP and GB-BP or $1 / \alpha 0$ or $1 / \alpha 1$ for GB-MMBP-2 and BLUE-MMBP-2) and packet processing and service time has a mean of $1 / \beta$.

The simulation environment based in a single router buffer that use First-In-First-Out (FIFO) scheduling. Subsequently, two states has been used for modeling the arrival packet in GB-MMBP-2 and BLUE-MMBP-2 as seen at Fig. 4, whereas a single state modeling is used in GB-BP and BLUE-BP as seen in Fig. 4 and 5 shows that GB starts dropping packets in early stage before the number of arrived packet reach the threshold.

\section{Performance Evaluation Results}

In this section, the performance for GB-MMBP-2, GB-BP, BLUE-MMBP and BLUE-BP is evaluated. This evaluation attempts to finds out which algorithm gets more satisfactory performance in both BP and MMBP-2. In other words if the traffic include the features of bursty and correlated by using MMBP-2 or without this features by using BP.

\section{Parameter Setting for the Compared Algorithms}

The parameters setting for all simulated algorithm are given in Table 2. The size of router buffer is chosen to be 20 in order to force the occurrence of a congestion rottenly (Abdel-Jaber et al., 2007a). The total number of slot is 2.000 .0000 , which is sufficient to give a precise results. The initDP value was set to 0.05 as recommended at original BLUE.

The packet arrival probability for state 1 in GBMMBP-2, GB-BP, BLUE-MMBP-2 and BLUE-BP is set to various values $(0.18-0.93)$ in order to cover all situation for the router buffer include no congestion, light congestion and heavy congestion. As such, the packet arrival interval is set as greater than and less than the value of the packet departure. Thus, no congestion will occur with values of packet arrival probability, for state 2 in GBMMBP-2 and BLUE-MMBP-2, less than 0.5 to reduce the number of arriving packets (Guan et al., 2007).

In case of MMBP-2 (GB-MMBP-2 and BLUEMMBP-2), which has two states, the transition probability between state 1 and state 2 is set to 0.1 and the probability that the next packet will come to the same state is 0.9 .

The remaining parameters (i.e., threshold, Dmax, Pinc andiniti DP) are set to values as provided in the BLUE algorithm (Feng et al., 2002).

\section{Performance Measures}

\section{Mean Queue Length, Throughput and Average Queuing Delay}

The performance measures, mql, $\mathrm{T}$ and $\mathrm{D}$ for BLUEMMBP-2, BLUE-BP, GB-BP and GB-MMBP-2, with various probabilities of packet arrival, are showed in Fig. 6-8. The measure mql represents the average number of packets at the router buffer at any time. The measure mql is calculated as the sum of product for each queue state with its probability as shown in "Equation 3":

$$
\mathrm{mql}=\sum_{i=0}^{k} i \times p_{i}
$$

where, $N$, is the finite buffer capacity (Woodward, 1994).

In order to avoid increasing the number of packet at the router buffer as a result of increasing the possibility of the congestion, mql has to be as small as possible. $\mathrm{D}$ is defined as the average waiting time for packets at the router buffer before get serviced. D is calculated using Little's law by dividing mql by $\mathrm{T}$ as shown in "Equation 4" (Woodward, 1994):

$D=\frac{m q l}{T}$

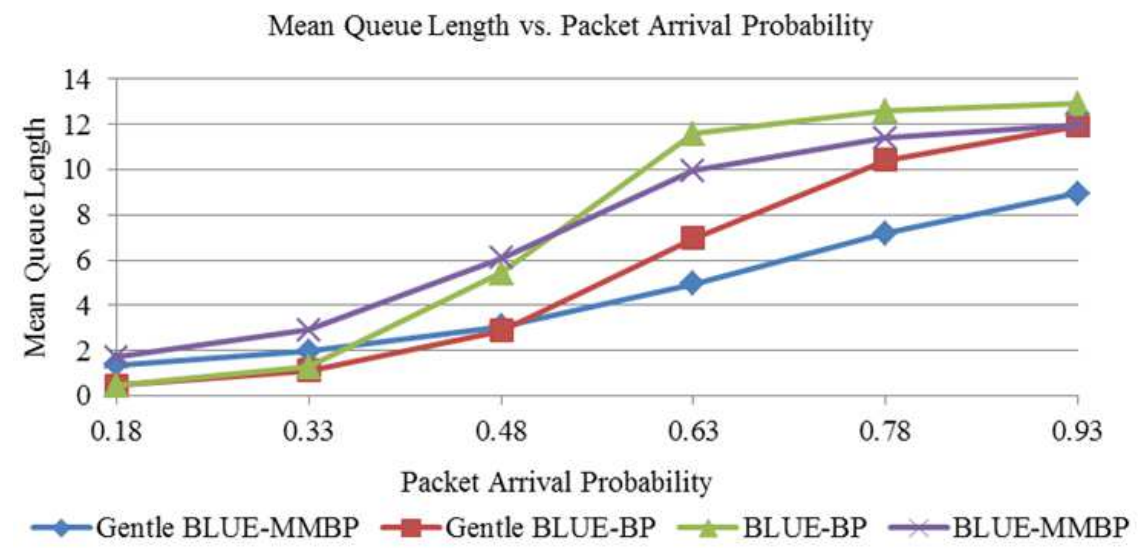

Fig. 6. mql Vs. packet arrival probability 


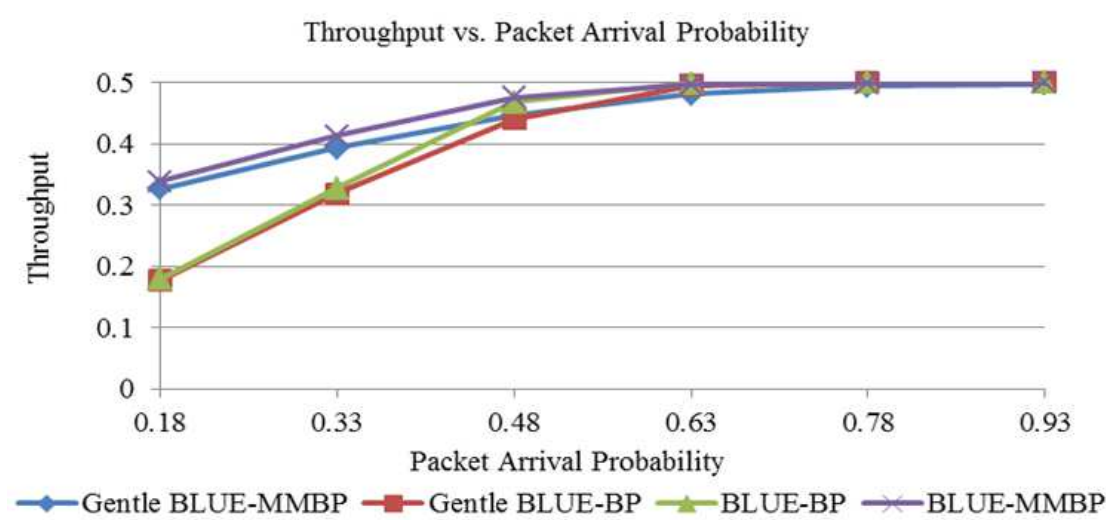

Fig. 7. T Vs. packet arrival probability

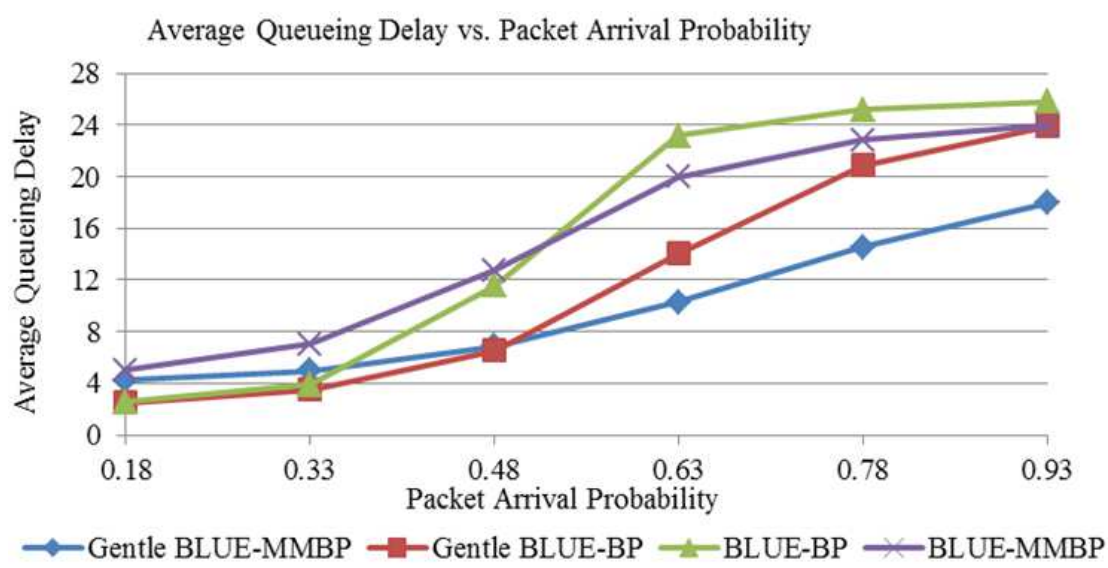

Fig. 8. D Vs. packet arrival probability

Table 2. Parameter settings

\begin{tabular}{|c|c|c|c|c|}
\hline \multirow[b]{2}{*}{ Parameter } & \multicolumn{4}{|l|}{ Methods } \\
\hline & BLUE & BLUE-MMBP-2 & $\begin{array}{l}\text { GENTLE } \\
\text { BLUE-BP }\end{array}$ & $\begin{array}{l}\text { GENTLE } \\
\text { BLUE-MMBP-2 }\end{array}$ \\
\hline Probability of packet arrival for state $1(\alpha 0)$ & $0.18-0.93$ & $0.18-0.93$ & $0.18-0.93$ & $0.18-0.93$ \\
\hline Probability of packet arrival for state $2(\alpha 1)$ & ----- & 0.5 & ----- & 0.5 \\
\hline Probability that the next packet remains in state 1 & ------ & 0.9 & ------ & 0.9 \\
\hline Probability that the next packet remains in state 2 & ------ & 0.9 & ------ & 0.9 \\
\hline Transition probability from state 1 and state 2 & ----- & 0.1 & ----- & 0.1 \\
\hline Transition probability from state 2 and state 1 & ----- & 0.1 & ----- & 0.1 \\
\hline Probability Packet departure $(\beta)$ & 0.5 & 0.5 & 0.5 & 0.5 \\
\hline Router buffer capacity & 20 & 20 & 20 & 20 \\
\hline Number of slots & 2000000 & 2000000 & 2000000 & 2000000 \\
\hline Threshold & 12 & 12 & 12 & 12 \\
\hline Freeze time & 0.01 & 0.01 & ----- & ----- \\
\hline Pin & 0.00025 & 0.00025 & ----- & ----- \\
\hline $\mathrm{P} d e$ & 0.000025 & 0.000025 & ----- & ----- \\
\hline InitDP & 0.05 & 0.05 & 0.05 & 0.05 \\
\hline
\end{tabular}

Figure 6 and 8 represent the value of mql and D for the compared algorithms. GB-BP and BLUE-BP provide more satisfactory performance comparing with the other algorithm. All algorithms provide less mql and D compared to BLUE-MMBP-2, when the packet arrival probability value is up to 0.33 (in case of no congestion) that is because the number of packets at the queue in GB-MMBP-2 is greater than GB-BP and BLUE-BP and all of them is less than those in BLUE-MMBP-2. By other means, because MMBP is transmitted to state 2 
(i.e., the packet arrival probability is changed to 0.5 ), which leads to increase the mql. GB-BP and GBMMBP-2 have the same mql and D values if the probability of packet arrival are between 0.33 and 0.48 . Both GB-BP and GB-MMBP-2 provide the same mql and $D$ results, because the probability of packet arrival for state 1 and state 2 in GB-MMBP-2 are almost equal to each other and are equal to packet arrival probability in GB-BP (close to 0.5 ). In addition, these results provide less $\mathrm{mql}$ and $\mathrm{D}$ performance measure compared to BLUE-BP and BLUE-MMBP-2 due to GB-BP and GBMMBP-2 drop more packets as given in Fig. 6 and 8 . GB-MMBP-2 provides more satisfactory and better mql and $\mathrm{D}$ performance measures compared to other algorithms when the packet arrival probability is greater than or equal because number of packets at the router buffer is less than the other algorithms in light and heavy congestion. GB-MMBP-2 is less than GB-BP in terms of D and mql, because GB-MMBP-2 has two states and the packets may arrived at state 2 so the number of arrived packet in state two in this stage is less than the number of arrived packet in state 1 so this reduce the number of packet at the router. GB-BP also provides better and lower mql and D measures compared with the other algorithm. In addition, BLUE-MMBP-2 offers less mql and $\mathrm{D}$ results compared with BLUE-BP.

$\mathrm{T}$ is the number of packet that successfully leave the router buffer after being served by the router. Figure 7 illustrates the T-based performance of all compared algorithms under various values of packet arrival probability. Figure 7 shows that BLUEMMBP-2 provides better $T$ results compared with the other methods. GB-MMBP-2 also provides better and higher $T$ results than GB-BP and BLUE-BP in case of no congestion (packet arrival probability is up to 0.33 ) because GB-BP and BLUE-BP serves less packets compared to algorithms and GB-MMBP-2 occupy the highest number of serves. The mql results at these arrival rates are higher for BLUE-MMBP-2 compared with the other algorithms, whereas those for GBMMBP-2 are higher than those for both GB-BP and BLUE-BP. Both GB-BP and BLUE-BP present similar $T$ results at these rates. When the value of packet arrival probability is above 0.33 , all the compared algorithms offer similar $\mathrm{T}$ results. These results are almost equal to the value of packet departure probability.

\section{Overflow PL Probability and Packet DP}

PL probability can be defined as the probability of losing packets after the router buffer get overflowed. Packet DP is the value of packet dropping probability that gives a good indicator about the network congestion status. The value of DP increases when there is a congestion and it give an accurate prediction before the buffer get overflowed.
A comparison between GB-MMBP-2, GB-BP, BLUE-MMBP-2 and BLUE-BP in term of PL and packet DP is shown in Fig. 9 and 10. The goal of the comparison is to evaluate which algorithm provide better performance measure in term of PL and DP.The amount of dropping packet before the router get overflowed using DP performance measure and after the packet get over flowed using PL.

Figure 9 shows that all the compared algorithms provide similar PL results when the packet arrival value is up to 0.48 because they overflow their router buffers at a similar frequency. All the compared algorithms, except for BLUE-BP provide similar PL results when the packet arrival value is between 0.48 and 0.63 (i.e., this range contains light congestion) because they overflow the router buffers at a frequency less than that for BLUE-BP. GB-MMBP-2 and GB-BP slightly yield the best and least PL performance in case of light and heavy congestion (the packet arrival probability is greater than or equal 0.63 ) because mql of GB-MMBP-2 is less than the other algorithm, so they overflow the router buffers at a frequency less than that for BLUE-MMBP-2 and BLUE-BP. BLUE-MMBP-2 loses fewer packets than BLUE-BP at these arrival probabilities.

Notably, Fig. 10 shows that BLUE-BP, BLUEMMBP-2 and GB-BP provide similar DP results when the value of packet arrival probability equals to 0.18 . The results of these methods are lower than the result of GB-MMBP-2 because MMBP can be transmitted to state 2 (i.e., the packet arrival probability is changed to 0.5 ), so the number of dropped packets is increased. When the arrival probability is 0.33 , BLUE-BP and BLUE-MMBP-2 drop the least number of packets and GB-BP drops fewer packets compared with GBMMBP-2. This scenario can be attributed to the queue length of GB-MMBP-2 being the largest (but not full) and that of GB-BP being larger than those of BLUE$\mathrm{BP}$ and BLUE-MMBP-2. If the arrival probability is equal to 0.48 , BLUE-BP and BLUE-MMBP-2 drop the least number of packets because of its queue length, but both GB-BP and GB-MMBP-2 drop similar numbers of packets because of the similar lengths of their queue buffers. When the value of packet arrival probability is 0.63 , GB-MMBP-2 drops the least number of packets among all the methods and BLUE-MMBP-2 drops fewer packets compared with GB-BP and BLUE-BP. In addition, GB-BP and BLUE-BP drop similar numbers of packets. When the arrival probability is larger than 0.63 (i.e., contains heavy congestion), GB-MMBP-2 and BLUE-MMBP-2 drop the lowest number of packets because their queue lengths are the smallest. GB-BP and BLUE-BP drop similar numbers of packets because they have similar queue lengths. 
Overflow Packet Loss Probability vs. Packet Arrival Probability

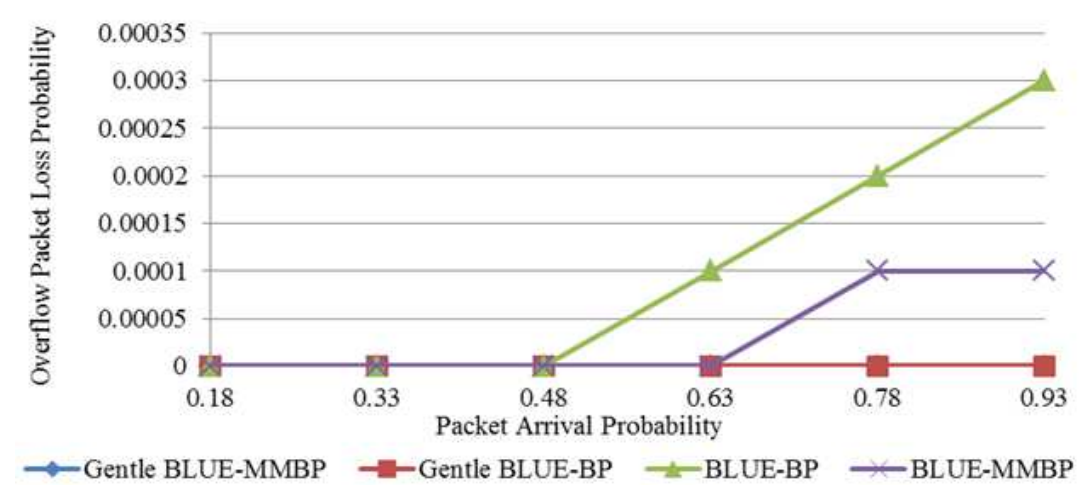

Fig. 9. Overflow PL probability Vs. packet arrival probability

Packet Dropping Probability vs. Packet Arrival Probability

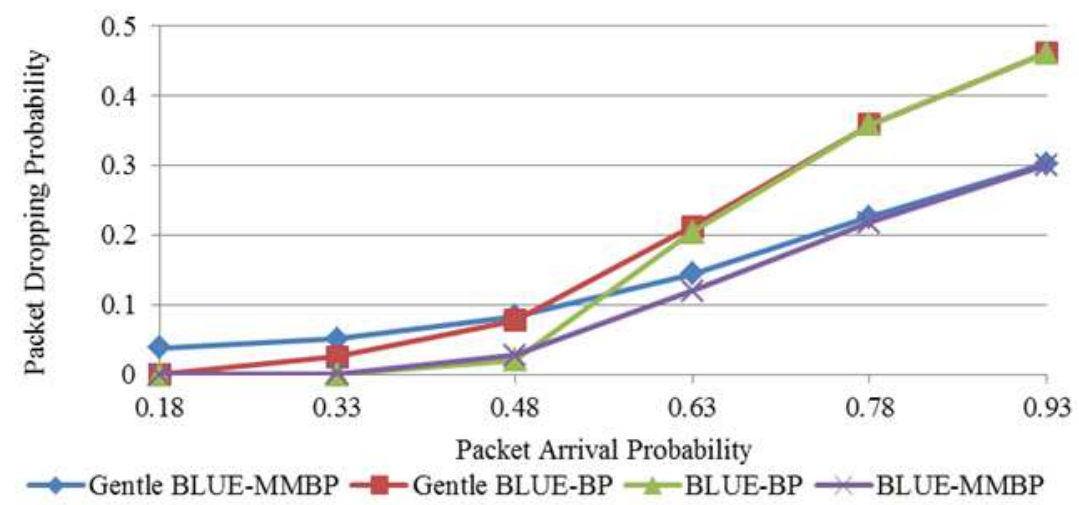

Fig. 12. Packet DP Vs. packet arrival probability

\section{Conclusion}

Planning network capacity plays a significant part in achieving QoS, so the evaluation of the AQM under some properties that exist in nature traffic has to be achieved. Internet traffic is aggregated by nature, aggregated traffic such as multimedia is bursty and correlated. The AQM evaluation process must consider the nature of the aggregated traffic by modeling traffics under burstiness and correlation.

Modeling traffic is an essential part of evaluating queue management methods. Queuing models such as $\mathrm{BP}, \mathrm{PP}$ and MMBP are used to validate or evaluate the performance of these AQM algorithms. The BP and PP fail to capture the properties of multimedia traffic, instead of that MMBP is the most widely used model for aggregated traffic with correlation and burstiness.

The results show that GB is better than the other algorithms. This condition implies that GB algorithm provides the best performance result in terms of mql, D, T, PL and DP compared with the other algorithms, especially when heavy congestion occurs.
In the future work, we intended to conduct analytical modeling of the queuing network to handle two queue nodes based on the priority policy using discrete time. Another analytical model can also be developed to handle $\mathrm{N}$ queue nodes.

\section{Acknowledgement}

We thankIslamic Science University of Malaysiaand Faculty of Science and Technology for supporting us.

\section{Funding Information}

The authors have no support or funding to report.

\section{Author's Contributions}

Adeeb Alsaaidah: Coordinate the paper from early to end, design the structure of research paper, write literature for the paper and conduct experiment and acquire data.

Mohd Zalisham: Design the research plan, help with the experiment and data collection and validate analysis for the paper. 
Mohd Fadzli: Help in designing the study and help to give input on the structure of the paper.

Hussein Abdel-Jaber: Verified the analysis and help to provide the input for the paper's structure.

\section{Ethics}

This article is original and contains unpublished material. The corresponding author confirms that all of the other authors have read and approved the manuscript and no ethical issues involved.

\section{References}

Abdel-Jaber, H., F. Thabtah and M. Woodward, 2015. Modelling discrete-time analytical models based on random early detection: Exponential and linear. Int. J. Model., Simulat. Sci. Comput. DOI: $10.1142 / \mathrm{S} 1793962315500282$

Abdel-Jaber, H., F. Thabtah, M. Woodward and W. Hadi, 2007a. Linear analysis for a BLUE congestion control algorithm using a discrete-time queue. Proceedings of the 3rd International Conference on Information Technology, (CIT' 07).

Abdel-Jaber, H., M.E. Woodward, F.A. Thabtah and M. Al-Diabat, 2007b. Modelling BLUE active queue management using discrete-time queue. Proceedings of the World Congress on Engineering, (WCE' 07), EBSCO Publishing, pp: 568-573.

Abdel-Jaber, H., M. Woodward, F. Thabtah and A. Abu-Ali, 2008. Performance evaluation for DRED discrete-time queueing network analytical model. J. Netw. Comput. Applic., 31: 750-770. DOI: $10.1016 /$ j.jnca.2007.09.003

Abdulkareem, M., K. Akil, A. Kalakech and S. Kadry, 2015. EFRED: Enhancement of fair random early detection algorithm. Int. J. Commun. Netw. Syst. Sci., 8: 282-294. DOI: 10.4236/ijens.2015.87028

Al-Diabat, M.O.F.L.E.H., H.U.S.S.E.I.N. Abdel-Jaber, F. Thabtah, O.S.M.A.N. Abou-Rabia and M. Kishta, 2012. Analytical models based discrete-time queueing for the congested network. Int. J. Model. Simulat. Sci. Comput., 3: 1150004-1150026 DOI: $0.1142 / \mathrm{S} 1793962311500048$

Alsaaidah, A., M. Zalisham, M. Fadzli and H. Abdel-Jaber, 2014. Gentle-BLUE: A new method for active queue management. Proceedings of the 3rd International Conference on Advanced Computer Science Applications and Technologies, Dec. 29-30, IEEE Xplore Press, Amman, pp:67-72. DOI: $10.1109 /$ ACSAT.2014.19

Baklizi, M., H. Abdel-Jaber, M.M. Abu-Alhaj, N. Abdullah and S. Ramadass et al., 2013. Dynamic stochastic early discovery: A new congestion control technique to improve networks performance. ICIC Int., 9: 1113-1126.
Baklizi, M., H. Abdel-Jaber, A.A. Abu-Shareha, M.M. Abualhaj and S. Ramadass, 2014. Fuzzy logic controller of gentle random early detection based on average queue length and delay rate. Int. J. Fuzzy Syst., 16: 9-19.

Feng, W.C., K.G. Shin, D.D. Kandlur and D. Saha, 2002. The blue active queue management algorithms. IEEE/ACM Trans. Netw., 10: 513-528. DOI: $10.1109 /$ TNET.2002.801399

Fischer, W. and K. Meier-Hellstern, 1993. The MarkovModulated Poisson Process (MMPP) cookbook. Performance Evaluat., 18: 149-171. DOI: 10.1016/0166-5316(93)90035-S

Floyd, S. and V. Jacobson, 1993. Random early detection gateways for congestion avoidance. IEEE/ACM Trans. Netw., 1: 397-413. DOI: 10.1109/90.251892

Floyd, S., R. Gummadi and S. Shenker, 2001. Adaptive RED: An algorithm for increasing the robustness of RED's active queue management.

Ghoreishi, S.E., A. Hamid Aghvami and H. Saki, 2015. Active queue management for congestion avoidance in multimedia streaming. Proceedings of the European Conference on Networks and Communications, Jun. 29-Jul. 2, IEEE Xplore Press,Paris, pp: 487-491. DOI: 10.1109/EuCNC.2015.7194123

Guan, L., I.U. Awan and M.E. Woodward, 2004a. Stochastic modelling of random early detection based congestion control mechanism for bursty and correlated traffic. IEE Proc. Software, 151: 240-247. DOI: 10.1049/ip-sen:20041089

Guan, L., M.E. Woodward and I.U. Awan, 2004b. Stochastic approach for modeling multi-class congestion control mechanisms based on RED in $\mathrm{TCP} / \mathrm{IP}$ networks.

Guan, L., M.E. Woodward and I.U. Awan, 2004c. Stochastic modelling of maintaining specified QoS constraints in discrete-time domain. Proceedings of the 13th International Conference on Computer Communications and Networks, Oct. 11-13, IEEE Xplore Press, pp: 109-114. DOI: 10.1109/ICCCN.2004.1401601

Guan, L., I.U. Awan, M.E. Woodward and X. Wang, 2007. Discrete-time performance analysis of a congestion control mechanism based on RED under multi-class bursty and correlated traffic. J. Syst. Software, 80: 1716-1725. DOI: 10.1016/j.jss.2006.12.549

Guan, L., M.E. Woodward and I.U. Awan, 2006. Control of queueing delay in a buffer with time-varying arrival rate. J. Comput. Syst. Sci., 72: 1238-1248. DOI: $10.1016 /$ j.jcss.2005.12.005

Kim, H.J., J.C. Shim, H.S. Kim, K.S. Cho and S.G. Choi, 2010. An Active Queue Management for QoS Guarantee of the High Priority Service Class. In: Communication and Networking, Kim, T., A.C.C. Chang, M. Li, C. Rong and C.Z. Patrikakis et al. (Eds.), Springer Berlin Heidelberg, pp: 37-45. 
Lee, M.H., Y.S. Mun and B.G. Kim, 1997. Performance analysis of delay-loss priority mechanism using Markov modulated arrival stream. IEE Proc. Commun., 144: 311-315. DOI: $10.1049 /$ ip-com:19971508

Lim, L.B., L. Guan, A. Grigg, I.W. Phillips and X.G. Wang et al., 2010. RED and WRED performance analysis based on superposition of $\mathrm{N}$ MMBP arrival proccess. Proceedings of the 24th IEEE International Conference on Advanced Information Networking and Applications, Apr. 20-23, IEEE Xplore Press, Perth, WA, pp:66-73. DOI: 10.1109 /AINA.2010.85

Lim, L.B., L. Guan, A. Grigg, I.W. Phillips and X.G. Wang et al., 2011. Controlling mean queuing delay under multi-class bursty and correlated traffic. J. Comput. Syst. Sci., 77: 898-916. DOI: $10.1016 /$ j.jcss.2010.08.007

Lim, L.B., L. Guan, A. Grigg, I.W. Phillips and X.G. Wang et al., 2009. Bounding queuing delay in a router based on superposition of N MMBP arrival process. Proceedings of the 18th International Conference on Computer Communications and Networks, Aug. 3-6, IEEE Xplore Press, San Francisco, CA, pp: 1-6. DOI: $10.1109 /$ ICCCN.2009.5235242

Liu, K.H., X. Ling, X.S. Shen and J.W. Mark, 2008. Performance analysis of prioritized MAC in UWB WPAN with bursty multimedia traffic. IEEE Trans. Vehicular Technol., 57: 2462-2473.

DOI: 10.1109/TVT.2007.912139
Ng, C.H., L. Yuan, W. Fu and L. Zhang, 1999. Methodology for traffic modeling using two-state Markov-modulated Bernoulli process. Comput. Commun., 22: 1266-1273. DOI: $10.1016 / \mathrm{S} 0140-3664(99) 00114-0$

Reddy, T.B. and A. Ahammed, 2008. Performance comparison of active queue management techniques. J. Comput. Sci., 4: 1020-1023.

Ryu, S., C. Rump and C. Qiao, 2003. Advances in internet congestion control. IEEE Commun. Surveys Tutorials, 5: 28-39. DOI: 10.1109/COMST.2003.5342228

Saaidah, A.M., M.Z. Jali, M.F. Marhusin and H. Abdel-Jaber, 2014. Markov-modulated Bernoullibased performance analysis for BLUE algorithm under bursty and correlated traffics. Proceedings of the International Conference on Computer, Communications and Control Technology, Sept. 2-4, IEEE Xplore Press, Langkawi, pp: 9-14. DOI: 10.1109/I4CT.2014.6914136

Samorodnitsky, G., 2007. Long range dependence. Foundat. Trends ${ }^{\circledR}$ Stochastic Syst., 1: 163-257. DOI: 10.1561/0900000004

Woodward, M.E., 1994. Communication and Computer Networks: Modelling with Discrete-Time Queues. 1st Edn., IEEE Computer Society Press, Hoboken, NJ, ISBN-10: 0818651725, pp: 205.

Zhou, W.H. and A.H. Wang, 2008. Discrete-time queue with Bernoulli bursty source arrival and generally distributed service times. Applied Math.Modell., 32: 2233-2240. DOI: 10.1016/j.apm.2007.07.014 